\title{
Group chase and escape with prey's anti-attack behavior
}

\author{
Shuai Zhang ${ }^{\mathrm{a}, *}$, Mingyong Liu ${ }^{\mathrm{a}}$, Xiaokang Lei ${ }^{\mathrm{b}, \mathrm{c}}$, Panpan Yang ${ }^{\mathrm{d}}$, Yunke Huang ${ }^{\mathrm{a}}$, Ruaridh Clark ${ }^{\mathrm{e}}$ \\ ${ }^{a}$ School of Marine Science and Technology, Northwestern Polytechnical University, Xi'an, Shaanxi, P. R. China \\ ${ }^{b}$ School of Information and Control Engineering, Xi'an University of Architecture and Technology, Xi'an, Shaanxi, P. R. China \\ ${ }^{c}$ KLINNS Lab, Xi'an Jiaotong University, Xi'an, Shaanxi, P. R. China \\ ${ }^{d}$ School of Electronic and Control Engineering,Chang'an University, Xi'an, China \\ ${ }^{e}$ Department of Mechanical and Aerospace Engineering, University of Strathclyde, Glasgow, UK
}

\begin{abstract}
Group chase and escape is widely observed in nature, where the predators approach the prey and the prey try to escape. An interesting phenomena occurs when a prey group is under attack, whereby some individuals perform anti-attack behavior that places themselves at a greater risks of being caught. It remains unclear why certain prey would risk their survival and what conditions and internal mechanisms trigger this anti-attack response. Using a set of local interaction rules among prey and predators, we proposed a continuous-space and discrete-time model that incorporates energy level, variable speed and handling time by considering different aggregation preferences of prey. We found that anti-attack behavior contributes to enhance the survivability of the prey group and the effect is more efficient in the presence of aggregation preference. The survivability can be improved if the fleeing prey have no aggregation preference while the anti-attack prey use a general aggregation preference.
\end{abstract}

Keywords: prey-predator, anti-attack behavior, group chase and escape, aggregation

\section{Introduction}

Systems containing prey and predators are commonly referred to as group chase and escape. Such systems have attract- ${ }_{30}$ ed significant attention recently $[1,2,3,4,5]$. Chase and escape 5 is an exciting example of collective behaviour that is widely observed in nature, with coyotes, elk and wolves in Yellowstone National Park [6, 7], wild chimpanzees in the Tai National Park [8], cooperative hunting in harris' hawks [9], subsocial spiders ${ }_{35}$ [10], flocks of birds [11] and schools of fish [12]. Different as-

10 pects of this prey-predator system have been studied using an agent-based model, such as leader election [13], pattern formation [14], adaptive collective behaviour [15], and social structure [16]. These works improve our understanding of how indi- ${ }_{40}$ viduals determine their future actions based on either their local 15 interactions or knowledge of the behavioral traits of either the predator or prey [17].

In the basic model by Kamimura and Ohira [1], the predators target the nearest prey, while the prey attempt to escape their nearest predators, on a two-dimensional lattice with periodic 20 boundary conditions. They found that there exists an optimal number of predators for a given number of prey to minimize the cost of catching all prey. Since then, many modified versions of the lattice model were proposed. From the perspective of catching efficiency, adding interaction among predators 25 (keep enough space among predators) [18], adding lazy or fast predators [19, 20], and using a collective chasing strategy [2]

${ }^{*}$ Corresponding author at: School of Marine Science and Technology, ${ }^{5}$ Northwestern Polytechnical University, China

Email address: shuaizhang@mail .nwpu.edu.cn (Shuai Zhang) can enhance the predators' catching efficiency. From the perspective of the prey's survivability, many papers have considered how aggregation behavior can produce a survival benefit. For example, aggregation improves group vigilance (many eyes effect)[21], makes capturing an individual prey more difficult by confusing the predators (confusion effect), dilutes an individual's risk of being caught (dilution effect) [22], and enables an effective defence against the predators (unity effect) [23]. Nishi et al. [3] provides a unique perspective by considering group chase and escape where prey can be converted to a predator. This conversion can be related to the spread of rabies, by considering that the predators are infected prey and the prey are susceptible to the virus. They found that the conversion can substantially reduce the lifetime of the prey when a large number of prey are initially present. Sato [5] added a third species to the conventional group chase and escape scenario, whereby one species is both a predator of the first species and prey for the third species. They found that when all of the individuals move randomly, no species becomes extinct. If, however, individuals escape from their nearest predator and immediately chase their nearest prey, the extinction of a species occurs. Thus, they stated the extinction of species is caused by group chasing.

It is noteworthy that the previous studies are all based on a situation where an individual prey will only try to escape from its predators, but does not performs any anti-attack behaviour. In reality, prey under attack will often display anti-attack behaviour despite such behaviour placing that individuals survival at greater risk. Examples of anti-attack behaviour are seen in colonies, such as harvester ants [24, 25] and water striders [26] - where individuals sacrifice themselves for the safety of the colony. It is worth noting that individuals in these colonies have 


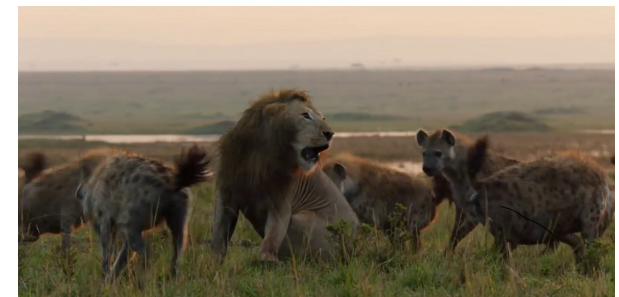

Figure 1: Illustration of anti-attack behaviour in hyenas.

a high genetic similarity, which means helping your colonymate is comparable to helping your own offspring. Examples of nti-attack behaviour are also seen in social animals where the genetic similarity is not as high. For example, painted wolves, known as cape hunting dogs in Africa, always live in packs. The individuals do display anti-attack behaviour when the pack is under attack by the lions, which places the anti-attack individuals survival at great risk but protects the puppies or the younger members. The same situation also occurs in hyenas ${ }_{120}$ (as illustrated in Fig. 1).

We extended the model [27] of group chase and escape in this paper by posing the following question: "How do the dynamic70 S change if the prey can perform anti-attack behaviour, instead of always escaping?" To answer this question, we proposed a continuous-space and discrete-time model that incorporates ${ }_{12}$ energy level, variable speed and handling time to investigate how the survival rate of prey changes as we vary the possibility 75 of anti-attack behaviour being displayed. The effect of prey's aggregation preference was also studied. We found that an individuals' anti-attack behaviour could enhance the group's survivability and this effect is more pronounced in the presence of ${ }_{130}$ aggregation preference.

\section{2. The model}

Based on the original group chase and escape model [27], we proposed a group chase, escape and anti-attack model by considering energy level, variable speed and handling time. The predators' maximum energy is higher but there are fewer preda- ${ }^{13}$ tors than prey. The predator has a speed advantage over the prey. The predator has a global view, while the prey has a local view. All predators need a period of time (including time spent on eating, and digesting captured prey $[28,29])$ to handle their captured prey.

The predator hunts the prey during the whole predation period, while each prey has two alternative behaviours: escape or anti-attack. Each prey/predator is labeled by an energy level, which determines its movement speed and living status (alive or dead). The energy of both predator and prey decline while energy by stopping all motion (animals feed on grass) or win-145 ing the anti-attack game (the details of the anti-attack game are given later), while the predator only obtains energy from its captured prey. The prey die if they are caught by a predator of a predator, which is a circle of radius $r_{\text {die }}>0$ ), while the predator may die because of losing the anti-attack game. The dead prey and predators are eliminated from the arena.

\subsection{Anti-attack game}

The specific forms of anti-attack game may vary with different species, and the consequence depends on many factors, such as speed, acceleration, manoeuvrability and muscle fibre power [30]. For simplicity, the anti-attack game is abstracted into a game of energy (strength) confrontation, where the side with advantage of energy will win the game. There are two phenotypes of prey: escape prey and anti-attack prey, which are pre-decided at the beginning of each simulation with a given ratio. The anti-attack game is triggered when the prey is close to the predator but not yet captured (a prey is located inside an area with radius $r_{\text {att }}>r_{\text {die }}$ centered on the predator). This game has two possible outcomes that are dependent on the energy levels of the two opponents: 1) The anti-attack prey win the game if the total energy of anti-attack prey in the attack area is higher than that of the predator, 2) Otherwise, the predator wins the game. The winning anti-attack prey divide the predators' current energy equally, or the winning predator inherits the captured prey's current energy. All losing individuals will be eliminated from the arena.

Remark 1. In animal world, the prey-predator relationship is not rigid. The prey can also feed on the predator if they have the opportunity. For example, the painted dogs and the hyenas can feed on each other. The prey divide the predator's energy as the payback to the risk in this paper.

\subsection{Energy update function}

Energy is the foundation of maintaining animals' movements. The role of internal energy reserves with regards to prey avoiding predators has been studied on multiple occasions $[31,32,33]$. The increase in energy per unit time is typically described by [31] as

$$
\gamma(u, x)=a(x) u-b(x)
$$

where $0 \leq u \leq 1$ represents the proportion of time that an animal spends feeding, and $x$ is energy reserves. If expenditure is independent of $u, b(x)$ is the mass-dependent metabolic rate and $a(x)$ is the rate at which energy is gained per unit time spent feeding. The model also assumes that $b(x)$ increases with energy reserves, and $a(x)$ either is constant or decreases with $x$. When $x$ corresponds to the body mass of a growing organism, $a(x)$ is likely to be an increasing function of $x$.

In our model, the energy update function is modified from the equation (1) as:

$$
\Delta e(\xi, v)=\xi a(\cdot)-(1-\xi) b(v)
$$

where $\xi=[0,1]$ is a switch function. It is a parameter that does not evolve with time but evolves with event, i.e. the value of parameter $\xi$ is achieved by event-triggering mechanism. For the prey, the escape behavior leads to $\xi=0$ while feed behavior leads to $\xi=1$. For the predator, the chase behavior leads 
150 to $\xi=0$ while feed behavior leads to $\xi=1 . a(\cdot)$ is the rate at which energy is gained per unit time spent feeding. For the prey, $a(\cdot)=\frac{U}{n_{\mathrm{p}}}+\frac{e_{\text {cha }}}{n_{\mathrm{a}}}$, where $U$ is the rate of energy acquisition in threat-free environment and $n_{p}$ is the number of neighbours 190 of the prey. $e_{\text {cha }}$ is the residual energy before the losing predator is dead, and $n_{a}$ is the number of participating anti-attack prey. $n_{p}$ and $n_{a}$ are introduced to simulate the resource competition. For the predators, $a(\cdot)=\sum e_{\operatorname{tar}}$, where $e_{\operatorname{tar}}$ contains the energy of captured prey (escape and anti-attack prey). It is reasonable 195 to assume that the energy dissipation rate of a prey/predator is speed-dependent. That is a slower speed burns energy slowly, while a higher speed burns energy more quickly, therefore $b(v)=k v^{\varphi}(k>0, \varphi>0)$ is described as an energy loss function for both prey and predators ${ }^{1}$.

The energy update functions for prey and predators are presented as follows:

$$
\begin{aligned}
& \Delta e_{\mathrm{tar}}(\xi, v)=\xi\left(\frac{U}{n_{p}}+\frac{e_{\mathrm{cha}}}{n_{a}}\right)-(1-\xi) k v^{\varphi} \\
& \Delta e_{\mathrm{cha}}(\xi, v)=\xi \sum e_{\mathrm{tar}}-(1-\xi) k v^{\varphi}
\end{aligned}
$$

\subsection{Kinematics equations}

We expanded Angelani's agent-based model by considering energy level, variable speed and handling time to study the group chase and escape with prey's anti-attack behavior [27]. For a prey, its position and velocity are updated according to

$$
\begin{aligned}
& \mathbf{r}_{i}(t+\Delta t)=\mathbf{r}_{i}(t)+\mathbf{v}_{i}(t+\Delta t) \Delta t \\
& \mathbf{v}_{i}(t+\Delta t)=v_{i}(t) \mathfrak{R}_{\eta}\left[\hat{\mathbf{d}}_{i}(t)\right]
\end{aligned}
$$

where $v_{i}(t)$ is the velocity modulus of prey $i$ and $\hat{\mathbf{d}}_{i}(t)$ is a unit vector describing the desired direction of prey $i$ in the next step. In [27], noise is added via an operator only to the alignment term as in the Vicsek model. Here, the same operator $\mathfrak{R}_{\eta}[\cdot]$ is used, but is simulated by rotating the final desired direction for ${ }^{20}$ a random angle uniformly distributed in the interval $[-\eta \pi, \eta \pi]$ with $\eta \in[0,1]$.

The speed of a prey/predator is energy-dependent instead of constant with it increasing with energy level but limited to a maximum speed. A sigmoid speed-energy function is intro-210 duced to describe this monotonically increasing relationship between the speed and the energy level as

$$
v(t)=\frac{V_{\max }}{1+\exp (-a(e(t)-c))}
$$

where $e(t)$ is the energy level at time $t(0 \leq e(t) \leq 1$ for each prey and $0 \leq e(t) \leq 3$ for each predator), $V_{\max }$ is the maximum speed, and $a, c>0$ are control parameters, see Fig. 2. $c$ is set in this paper at half of the maximum energy reserve for an

${ }^{1} b(v)=k v^{\varphi},(k>0, \varphi>0)$ is a reasonable energy loss function derived from physical systems. $k$ is a flexible coefficient of energy loss satisfying $k \ll U$. We investigate exponent $\varphi=0.5,1,2,3,4$ for the internal energy loss function of living beings. The results indicate that the findings are robust against the value of the exponent. agent, where $e(t)=c$ denotes the energy point where function's gradient is maximum. The speed increases with the energy level of an agent from zero to the maximum value. The results of the model is robust against the parameter $a$ when the parameter $c$ is the half of the maximum energy reserve.

The desired direction $\mathbf{d}_{i}(t)$ considers the aggregation preference among prey, pursuit-evasion relationship between predator-prey, and an agent's interaction with its environment. It contains four components: aliment effect $\mathbf{f}_{i}^{\mathrm{vel}}$ and position adjustment $\mathbf{f}_{i}^{\text {pos }}$ (which are responsible for aggregation preference), escape interaction $\mathbf{f}_{i}^{\text {esp }}$ and environmental constraint $\mathbf{f}_{i}^{\text {wall }}$,

$$
\begin{aligned}
& \mathbf{d}_{i}(t)=\hat{\mathbf{f}}_{i}^{\mathrm{vel}}+\beta \hat{\mathbf{f}}_{i}^{\mathrm{pos}}+\gamma \hat{\mathbf{f}}_{i}^{\mathrm{esp}}+\mathbf{f}_{i}^{\mathrm{wall}} \\
& \mathbf{f}_{i}^{\mathrm{vel}}=\sum_{j \in S_{i}^{\mathrm{vel}}} \mathbf{v}_{j} \\
& \mathbf{f}_{i}^{\mathrm{pos}}=\sum_{j \in S_{i}^{\mathrm{pos}}}\left(\frac{1}{\left\|\mathbf{r}_{i}-\mathbf{r}_{j}\right\|}-\frac{r_{0}}{\left\|\mathbf{r}_{i}-\mathbf{r}_{j}\right\|^{3}}\right) \hat{\mathbf{r}}_{i j} \\
& \mathbf{f}_{i}^{\mathrm{esp}}=z_{i} \sum_{j \in S_{i}^{\text {esp }}}\left\|\mathbf{r}_{i}-\mathbf{r}_{j}\right\|^{-2} \hat{\mathbf{r}}_{i j} \\
& \mathbf{f}_{i}^{\text {wall }}=s\left(v \frac{\mathbf{r}_{i}}{r_{i}}+\mathbf{v}_{i}\right) \\
& s=\left\{\begin{array}{lll}
0 & \text { if } & r_{i} \in\left[0, r_{a}\right] \\
-\frac{1}{2} \sin \left(\frac{\pi}{r_{\text {wall }}}\left(r_{i}-r_{a}\right)-\frac{\pi}{2}\right)-\frac{1}{2} & \text { if } & r_{i} \in\left[r_{a}, r_{a}+r_{\text {wall }}\right] \\
-1 & \text { if } & r_{i}>\left[r_{a}, r_{a}+r_{\text {wall }}\right]
\end{array}\right.
\end{aligned}
$$

where $\beta$ and $\gamma$ measure the relative strength of corresponding term. $\hat{\mathbf{r}}_{i j}$ is a unit vector from agent $j$ to agent $i . S_{i}^{\text {vel }}$ and $S_{i}^{\text {pos }}$ are the sets of aliment effect and position adjustment with the same limited aggregation range $r_{\mathrm{p}} . S_{i}^{\text {esp }}$ is the set of detected opponents with a limited perception range $r_{\mathrm{tar}} \geq r_{\mathrm{p}}$. The aggregation preference means the prey needs to update its states even when they are in threat-free environment. Here, $\mathbf{f}_{i}^{\mathrm{vel}}$ is responsible for synchronizing the velocities [34]. $\mathbf{f}_{i}^{\text {pos }}$ is responsible for aggregation and collision avoidance by adopting a long range attraction and short range repulsion function (other similar functions are also applicative) [35], where $r_{0}=1$ is a predefined small constant which regulates the expected distance among preys. $\mathbf{f}_{i}^{\text {esp }}$ is responsible for intergroup interaction. Based on Angelani's work [27], a weighted average of neighboring opponents with a power law is used to describe this prey-predator interaction, where $z_{i}=1$ denotes that the prey attempts to escape from the predators (escape behaviour), and $z_{i}=-1$ denotes that the prey moves towards the predators (anti-attack behaviour). The environmental constraint $\mathbf{f}_{i}^{\text {wall }}$ is responsible for limiting the individuals within closed, soft boundaries [2] (for example, a closed-soft boundary could be a river, gully, bush, fence, highland or canyon that could block a direction of escape for prey), where $r_{\mathrm{a}} \gg 0$ is the radius of the circular arena, $r_{\text {wall }} \ll r_{\mathrm{a}}$ is the width of the boundary, $\mathbf{r}_{i}$ is the radial vector and $r_{i}=\left|\mathbf{r}_{i}\right|$.

For a predator, its update equations are similar to the prey in equation (4). However, the desired direction $\mathbf{d}_{i}(t)$ that captures the intra- and intergroup interacting force is limited to position 


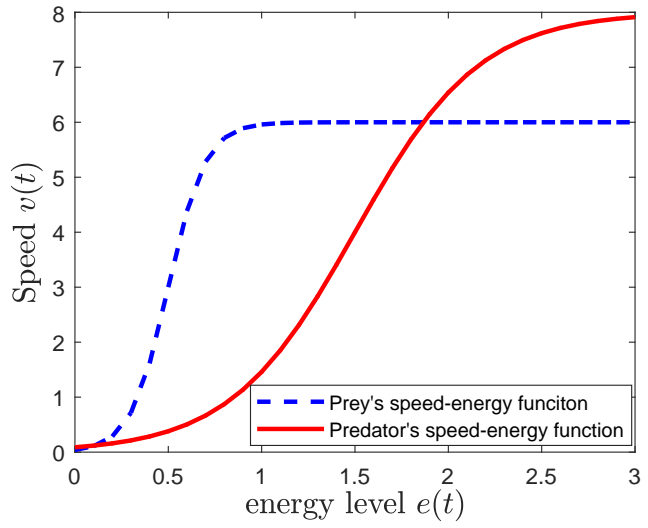

Figure 2: (Colour online) Sigmoid speed-energy functions of prey and predators. In order to have a successful predation, the predator has speed and energy advantages over the prey. The maximum/initial energy value for a prey is 1 (a reference energy level), while it is triple that for a predator. $V_{\max , \mathrm{t}}=6, a=10, c=0.5$ are used for prey. $V_{\max , \mathrm{c}}=8, a=3, c=1.5$ are used for predators.

adjustment, chase interaction and environmental constraint, as the cooperative hunting behavior of predators is not considered in the model. The position adjustment with short-range repulsion is needed because of individual space requirements and ${ }^{26}$ collision avoidance [36]. We assume that the predators try to chase the nearest prey (energy saving) while following a group of prey (intrinsic greedy), thus a power-law weighted average of nearby prey is also used for the predators $\left(\mathbf{f}_{i}^{\text {chs }}\right.$ is the same as $\mathbf{f}_{i}^{\text {esp }}$, but with $z_{i}=-1$ only, which means a predator always hunts the prey). The desired direction of a predator can be written as

$$
\mathbf{d}_{i}(t)=\beta \hat{\mathbf{f}}_{i}^{\mathrm{pos}}+\gamma \hat{\mathbf{f}}_{i}^{\mathrm{chs}}+\mathbf{f}_{i}^{\mathrm{wall}} .
$$

Note that during the predation event, the predator has to stop for a period of time $\tau$, which is regarded as handling time.

\section{Simulation}

At the beginning of each simulation, we place 10 predators and 100 prey at random locations inside a closed circular arena. The number of prey and predators is set for a scenario where there are a few strong predators and many weak prey. Further simulation parameters are shown in Table 1. See Fig. 3 for a 275 snapshot taken from a simulation run. More simulation footage M4.

We focus on the final state of this game model from the perspective of the prey, recording how many prey survived and how 280 long the prey survived before being caught. $T_{\text {tar }}$ is defined as the period from the start of the simulation to the time when the last prey was caught. A maximum simulation length $\left(T_{\max }=1000\right.$ timesteps, the results are robust and $T_{\max }$ is not critical when it is set large enough.) was applied, which was long enough for $\mathrm{a}_{285}$ stable state to emerge where one of the two groups was totally exterminated. For cases where some prey are alive at the end of the simulation, we assume that the entire lifetime of prey is at its maximum value as simulated prey are able to live forever

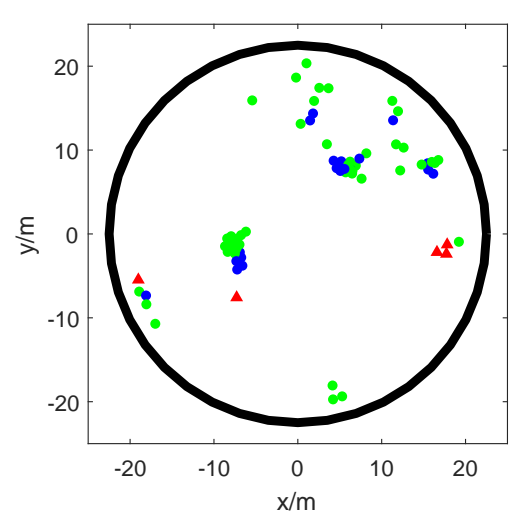

Figure 3: (Colour online) Snapshot of a group chase, escape and anti-attack configuration from the simulation run M1. The red triangles are predators, the green and blue circles are escape and anti-attack prey respectively.

if all the predators are dead. The average lifetime of the prey group, $T_{\mathrm{tar}}$, for $n$ runs is

$$
\bar{T}_{\mathrm{tar}}=\frac{n T_{\mathrm{tar}}+\left(n_{\mathrm{tot}}-n\right) T_{\mathrm{max}}}{n_{\mathrm{tot}}} .
$$

We also consider the portion of the prey group that survives. The average survival portion is

$$
\bar{S}_{\mathrm{tar}}=\frac{\sum S_{\mathrm{tar}}}{n_{\mathrm{tot}}} .
$$

The entire lifetime $T_{\mathrm{tar}}$, and the survival portion $S_{\text {tar }}$ were investigated for prey groups containing different portions $P$ of anti-attack prey. $P=0$ for no anti-attack prey in the group and $P=1$ for all prey using anti-attack behaviour.

\subsection{Effect of anti-attack}

We first analyzed the effect of anti-attack behaviour on group chase and escape where there was no aggregation preference for prey. The results in Fig. 4(a) and 4(b) show that the average lifetime $T_{\text {tar }}$ of the prey group has an overall upward trend as anti-attack possibility $P$ increases. This trend was independent of the values of $\varphi$ in the energy loss function $b(v)=k v^{\varphi}$, with $\varphi=2$ used in the following simulations. This conclusion is supported by the results of survival portion $S_{\text {tar }}$ of the prey, where the survival portion increases monotonously with antiattack possibility $P$. The results suggest that the optimal case for the prey is that the whole group uses anti-attack behaviour. Another result is that the lifetime decreases slightly with the number of anti-attack prey when the anti-attack prey start appearing, which indicates that a small number of anti-attack prey is detrimental to the survivability of the prey group.

The results in Fig. 4(c) and 4(d) show the survival results of two different phenotypes of prey. The lifetime and the survival portion of the anti-attack phenotype are both lower than that of the escape phenotype, which indicates that the anti-attack prey are exposed to a greater risk of capture than the escape prey. Another result is that there is no prey left alive at the end of the simulation until anti-attack portion $P>0.4$, which means a minimum proportion of anti-attack prey is needed for a prey 
Table 1: Model parameters.

\begin{tabular}{llll}
\hline Parameter & Value & Description & Range \\
\hline$\Delta t$ & 0.01 & length of one time step & $\Delta t>0$ \\
$r_{\mathrm{a}}$ & 20 & radius of circular arena & $r_{\mathrm{a}}>r_{\mathrm{p}} \gg 0$ \\
$r_{\text {wall }}$ & 0.5 & width of wall & $0<r_{\mathrm{wall}} \ll r_{\mathrm{a}}$ \\
$r_{\mathrm{tar}}$ & 10 & perception range of a prey (local view) & $r_{\mathrm{p}} \leq r_{\mathrm{tar}} \leq r_{\mathrm{a}}$ \\
$r_{\text {cha }}$ & 40 & perception range of a predator (global view) & $r_{\text {cha }}=2 r_{\mathrm{a}}$ \\
$r_{\mathrm{p}}$ & 5 & range of aggregation & $r_{\mathrm{att}}<r_{\mathrm{p}} \leq r_{\mathrm{tar}}$ \\
$r_{\text {die }}$ & 1 & radius of dead area & $r_{\mathrm{die}} \geq 0$ \\
$r_{\text {att }}$ & 3 & radius of attack area & $r_{\text {die }}<r_{\mathrm{att}}<r_{\mathrm{p}}$ \\
$\beta$ & 1 & strength of position adjustment & $\beta \geq 0$ \\
$\gamma$ & 1.2 & strength of chase-escape interaction & $\gamma \geq 0$ \\
$U$ & 0.01 & energy obtaining rate for a neighbourless prey & $0<U \ll 1$ \\
$k$ & 0.0001 & energy dissipation rate for a moving prey/predator & $0<k \ll U$ \\
$\eta$ & 0.1 & noise strength & $\eta \in[0,1]$ \\
$\tau$ & 10 & handling time (timestep) & $\tau \geq 0$ \\
\hline
\end{tabular}

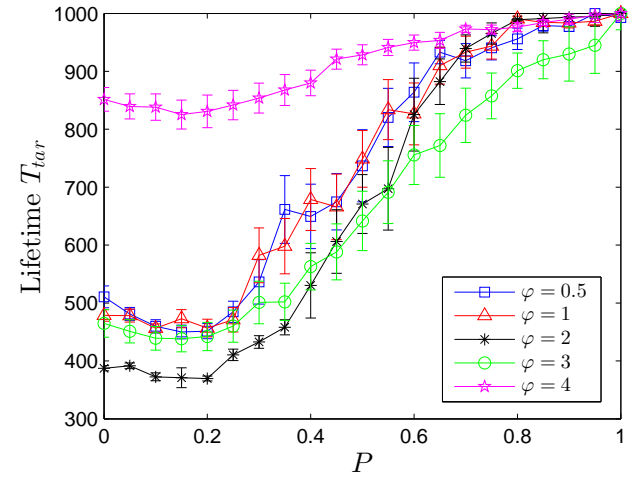

(a)

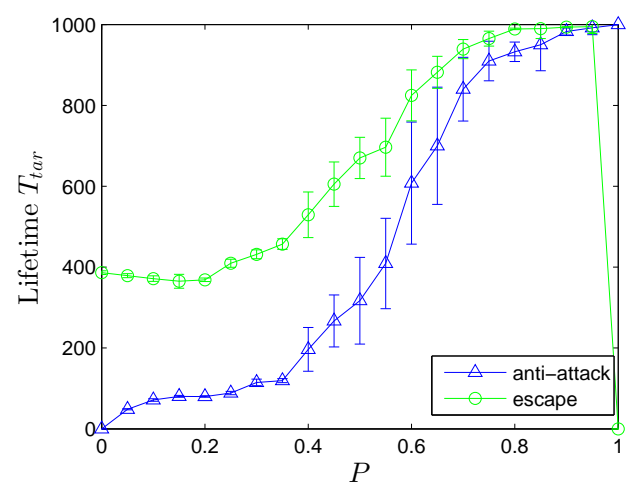

(c)

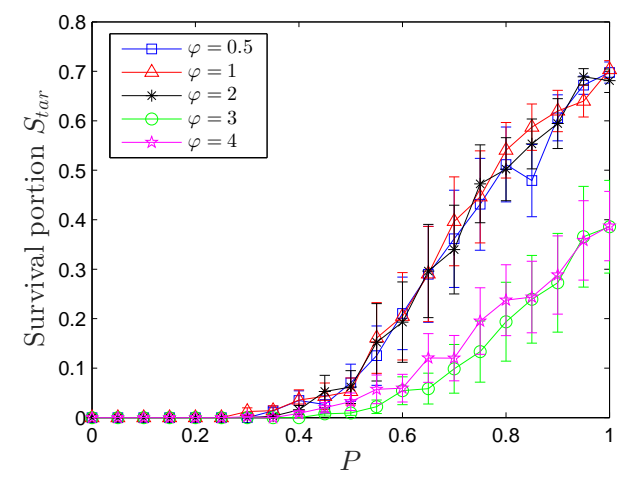

(b)

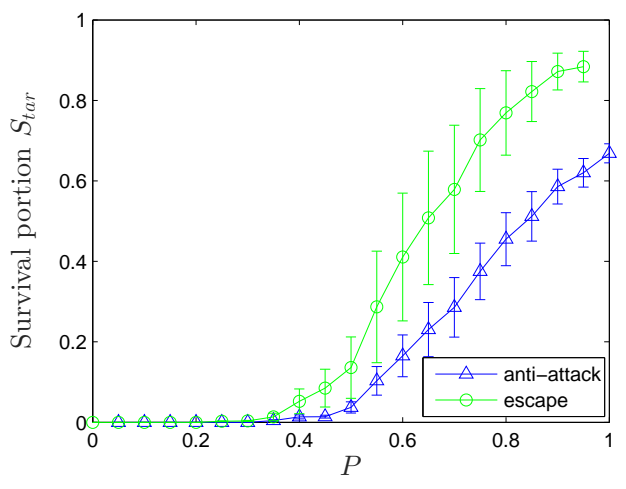

(d)

Figure 4: (Colour online) The survival results of prey with different anti-attack portion $P$. a) The lifetime $T_{\operatorname{tar}}$ of the whole group changing with anti-attack possibility $P$. b) The survival portion $S_{\text {tar }}$ of the whole group changing with anti-attack possibility $P$. c) The lifetime $T_{\text {tar }}$ of each phenotype changing with anti-attack possibility $P$. d) The survival portion $S_{\text {tar }}$ of each phenotype changing with anti-attack possibility $P . \varphi=2$. The results are obtained by 1000 independent runs. 
290 group to successfully avoid predation. These results indicate that it is not in the group's interest for individuals to act selfishly. An optimal strategy could be for the whole prey group to employ anti-attack behaviour, but this is not a stable strategy as anti-attack prey are at a greater risk of capture.

\subsection{Effect of aggregation}

Predators have shaped the evolution of aggregation behaviour, which is regarded as a good strategy for prey to avoid predation $[3,21,22,23]$. It is worth considering whether aggregation of living beings is motivated by improving their antiattack response and, hence, chances of survival? We now analyze the influence of aggregation preference for prey with antiattack behaviour in a group chase and escape simulation. For comparison, we also present the results when no aggregation preference is present for the prey.

305 The results in Fig. 5(a) show that the lifetime of prey $T_{\operatorname{tar}}$ under aggregation preference increases monotonically with antiattack portion $P$ until it reaches saturation at $P=0.5$. This indicates that the aggregation preference of prey can enhance their survival time and makes it easier for them to have a comthat the aggregation preference is not suitable in the situation where there are a relatively small number of anti-attack prey $(P \leq 0.1)$. From Fig. 5(b), we can obtain similar results that the aggregation preference has a positive effect on the survival 315 portion of the prey group as well. Another result is that the minimum threshold $P$ for achieving at least one survivor under aggregation preference is less than it is under no aggregation preference.

The main reason why aggregation preference produces an advantage, in most situations, in terms of lifetime and survival portion is that the aggregation behaviour increases the possibility that anti-attack prey defeat a predator. For an individual prey with no aggregation preference, it does not approach its neighbouring prey, leading to a smaller possibility of encoun- ${ }^{345}$ tering other anti-attack individuals during the predation game. In contrast, the anti-attack individuals with aggregation preference have a greater chance of accumulating enough energy to defeat a predator. When there is a relatively small number of anti-attack prey, aggregation is not always the best option as350 anti-attack prey are unlikely to gather in sufficient numbers to defeat the predator and will be captured every time they display anti-attack behavior.

\subsection{Aggregation strategy}

To improve the survivability of the whole prey group, we propose that the escape prey use no aggregation preference, while the anti-attack prey use an aggregation preference. We propose two specific aggregation tactics for the anti-attack prey: 1) 360 $_{36}$ General aggregation, in which an anti-attack prey has an aggregation preference to all its neighbours (including escape prey and anti-attack prey); 2) Selected aggregation, in which an antiattack prey has aggregation preference only to other anti-attack prey.

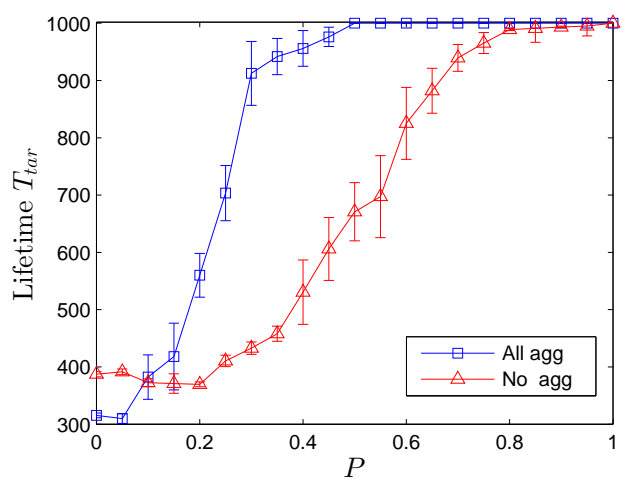

(a)

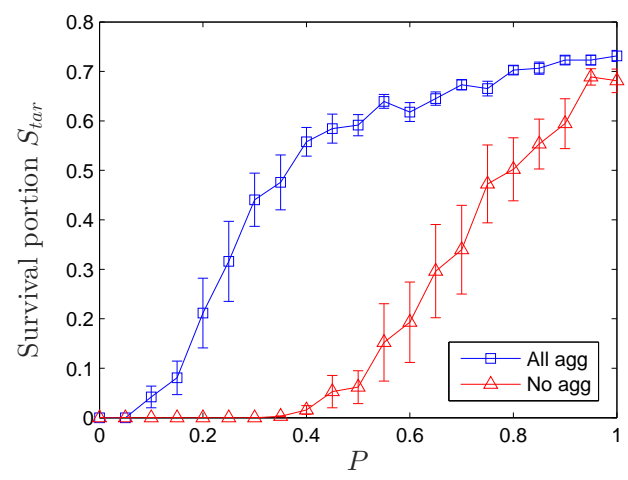

(b)

Figure 5: (Colour online) The survival results from all aggregation and no aggregation groups with different anti-attack portion $P$. a) The lifetime $T_{\operatorname{tar}}$ of prey from aggregation and no aggregation groups. b) The survival portion $S_{\text {tar }}$ of prey from aggregation and no aggregation groups. $\varphi=2$. The results are obtained by 1000 independent runs.

In Fig. 6, we show the survival results for prey under all aggregation preference, no aggregation preference, selected aggregation preference and general aggregation preference. The results indicate that when the escape prey use no aggregation preference, the best option for the anti-attack prey is to use general aggregation preference.

Intuition would suggest that the selected aggregation tactic should produce a similar or better result than the general aggregation strategy for prey. However, the general aggregation tactic obtains the best performance among the four different aggregation strategies in terms of lifetime and survival portion. The mechanism for such a situation can be explained as follows. If there is no anti-attack neighbour nearby to an antiattack individual, this individual using selected aggregation behavior is going to move towards the nearest predator, increasing the likelihood of meeting a predator early in the simulation before it has gathered with other anti-attack prey. This is similar to the case that the individual uses no aggregation preference. On the other hand, an anti-attack prey can keep alive if it uses the general aggregation strategy, as it is attracted to both type$s$ of prey increasing the likelihood of forming a group of prey before meeting a predator. This is partly due to the aggregation force counteracting the driving force of the predator. A larger 


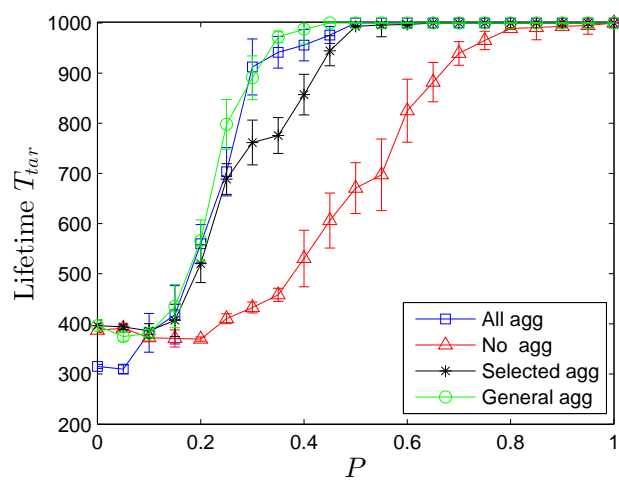

(a)

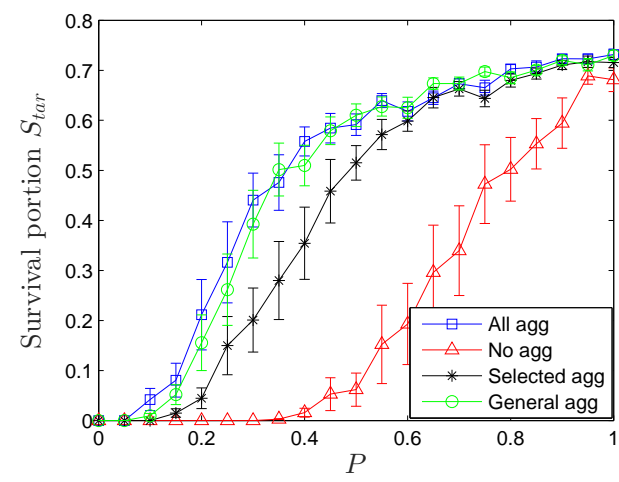

(b)

Figure 6: (Colour online) The survival results from four different situations. a) The lifetime $T_{t a r}$ of prey from four different situations. b) The survival portion $S_{t a r}$ of prey from four different situations. $\varphi=2$. The results are obtained by 400 1000 independent runs.

group of prey is more likely to contain multiple anti-attack prey that will increase the chances of success during the anti-attack game with the predator.

\subsection{Discussion}

The conclusions we obtained above are quite general, while quantitative details seem to be parameters-dependent. In this subsection, we elaborate on how parameters in Tab. 1 are chose and what is the effect of different values of each parameter on the results of the model. The parameters are generalized into energy parameters and special parameters.

The environmental parameters were chose in accordance with the reality as much as possible. These parameters contain ${ }_{415}$ $r_{a}, r_{\mathrm{wall}}, r_{\mathrm{tar}}, r_{\mathrm{cha}}, r_{p}, r_{\mathrm{die}}, r_{\mathrm{att}} . r_{a}$ is the radius of simulation arebility of a prey/predator, thus reducing the influence of boundary effect. $r_{\text {wall }}$, the width of the closed-soft boundary, should be much less than the radius of the arena. $r_{\mathrm{tar}}, r_{\mathrm{cha}}, r_{p}, r_{\mathrm{die}}, r_{\mathrm{att}}$ are perception range of prey/predator, aggregation range, dead ${ }_{420}$ range and attack range respectively, which were chose carefully for a real group chase and escape scenario. The environmental parameters can be chose with a great flexibility within the reasonable range, and they do not have important influence on the

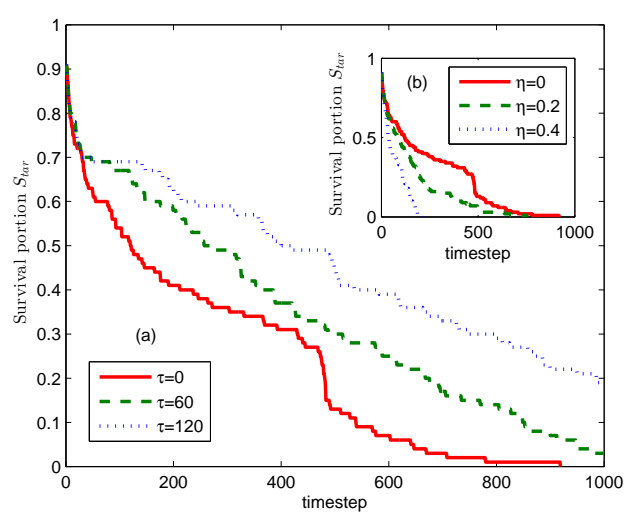

Figure 7: (Colour online) Survival portion of prey group with timesteps in a single run with $P=0.2$. (a) The effect of handling time $(\eta=0)$. (b) The effect of noise strength $(\tau=0)$.

results.

The motion parameters contain $\beta$ and $\gamma$, which together shape the motion strategy of a prey/predator. $\beta=1, \gamma=1.2$ were used for the reason that chase/escape is more important than aggregation preference and environmental constraint. The results of the model are similar if $\gamma$ is increased slightly. However, the modmental constraint and aggregation preference if $\gamma$ is increased significantly.

The energy parameters contain $U$ and $k$. $\mathrm{U}=0.01$ was given as energy obtaining rate for a neighborless prey, describing the speed of energy recovery for a prey when eating and having a rest. Obviously, it should be much less than the maximum energy level (the maximum energy level for prey is 1 as a reference value). $k=-0.0001$ was given as energy dissipation rate for a moving prey/predator. The energy dissipation rate must be far less than the energy obtaining rate as the period of activity of a prey/predator is commonly longer in comparison with the feeding period.

The special parameters contain $\eta$ and $\tau$. Analyzing the dynamics in the single runs, it is evident that the noise has the effect of decreasing the survival time, disturbing more the escape/anti-attack process of prey. The handling time of predator, provides a "peace time" for the prey, thus having the effect of increasing the survival time of prey. Fig.7 shows the effects of noise strength $\eta$ and handling time $\tau$.

\section{Conclusions}

In this paper, the agent-based model is used to investigate the problem of group chase and escape when prey exhibit antiattack behaviour. Anti-attack behaviour sets up an interesting situation, in which prey may defeat a predator or even the whole predator group through prey cooperation. By considering different aggregation strategies for prey, we found that antiattack behaviour can increase the survivability of a prey group, although it exposes the anti-attack prey to greater risks. The aggregation preference of prey enhances their chances of success 
425 when facing the predator in the anti-attack game and, hence, makes it more likely that the group executes a successful defense. However, it is not suitable to use all aggregation preference strategy in the situation where there are a relatively s- ${ }_{490}$ mall number of anti-attack prey. A more suitable aggregation 430 strategy for this scenario involves escape prey using no aggregation preference and anti-attack prey using the general aggregation preference. Our work highlights the role of anti-attack ${ }_{495}$ behaviour in the group chase and escape. Moreover, our work offers a deeper understanding of aggregation phenomena for animals that live in packs in order to improve their anti-attack response and, hence, chances of survival. One of the applica-500 tions of our work is artificial systems where incentivization of collective behaviour could provide a passive way to ensure individuals don't always act selfishly, such that we can implicitly affect the motivation of the individuals without organizing or $_{505}$ intervening with the individuals' preference.

\section{Acknowledgements}

This work was partially supported by the National Natural Science Foundation of China (NSFC) under Grants 51379176,

445 51679201, and Science and Technology on Underwater Information and Control Laboratory under Grants 6142218051806. 515

\section{References}

[1] A. Kamimura, T. Ohira, Group chase and escape, New Journal of Physics ${ }_{520}$ 12 (5) (2010) 053013

[2] M. Janosov, C. Virágh, G. Vásárhelyi, T. Vicsek, Group chasing tactics: how to catch a faster prey, New Journal of Physics 19 (5) (2017) 053003.

[3] R. Nishi, A. Kamimura, K. Nishinari, T. Ohira, Group chase and escape with conversion from targets to chasers, Physica A: Statistical Mechanics ${ }_{525}$ and its Applications 391 (1-2) (2012) 337-342.

[4] H. Wang, W. Han, J. Yang, Group chase and escape with sight-limited chasers, Physica A: Statistical Mechanics and its Applications 465 (2017) 34-39.

[5] M. Sato, Chasing and escaping by three groups of species, Physical Re- ${ }_{530}$ view E 85 (6) (2012) 066102.

[6] E. M. Gese, Territorial defense by coyotes (canis latrans) in yellowstone national park, wyoming: who, how, where, when, and why, Canadian Journal of Zoology 79 (6) (2001) 980-987.

[7] W. J. Ripple, E. J. Larsen, Historic aspen recruitment, elk, and wolves $\mathrm{in}_{535}$ northern yellowstone national park, usa, Biological Conservation 95 (3) (2000) 361-370.

[8] C. Boesch, H. Boesch, Hunting behavior of wild chimpanzees in the tai national park, American journal of physical anthropology 78 (4) (1989) $547-573$.

[9] J. C. Bednarz, Cooperative hunting in harris' hawks (parabuteo unicinctus), Science 239 (4847) (1988) 1525.

[10] K. W. Kim, B. Krafft, J. C. Choe, Cooperative prey capture by young subsocial spiders, Behavioral Ecology and Sociobiology 59 (1) (2005) $92-100$.

[11] G. M. Hilton, W. Cresswell, G. D. Ruxton, Intraflock variation in the speed of escape-flight response on attack by an avian predator, Behavioral Ecology 10 (4) (1999) 391-395.

[12] M. Zheng, Y. Kashimori, O. Hoshino, K. Fujita, T. Kambara, Behavior pattern (innate action) of individuals in fish schools generating efficient collective evasion from predation, Journal of Theoretical Biology 235 (2) (2005) 153-67.

[13] V. Karpov, I. Karpova, Leader election algorithms for static swarms, Biologically Inspired Cognitive Architectures 12 (2015) 54-64.

[14] H. Oh, A. R. Shirazi, C. Sun, Y. Jin, Bio-inspired self-organising multirobot pattern formation: A review, Robotics and Autonomous Systems 91 (2017) 83-100.
[15] S. Kernbach, D. Häbe, O. Kernbach, R. Thenius, G. Radspieler, T. Kimura, T. Schmickl, Adaptive collective decision-making in limited robot swarms without communication, The International Journal of Robotics Research 32 (1) (2013) 35-55.

[16] M. J. Dostie, D. Lusseau, T. Bonnell, P. M. R. Clarke, G. Chaplin, S. Kienzle, L. Barrett, S. P. Henzi, Proof of principle: the adaptive geometry of social foragers, Animal Behaviour 119 (2016) 173-178.

[17] S. H. Lee, H. K. Pak, T. S. Chon, Dynamics of prey-flock escaping behavior in response to predator's attack., Journal of Theoretical Biology 240 (2) (2006) 250-259.

[18] T. Saito, T. Nakamura, T. Ohira, Group chase and escape model with chasers interaction, Physica A: Statistical Mechanics and its Applications 447 (2016) 172-179.

[19] M. Masuko, T. Hiraoka, N. Ito, T. Shimada, The effect of laziness in group chase and escape, Journal of the Physical Society of Japan 86 (8) (2017) 085002 .

[20] T. Iwama, M. Sato, Group chase and escape with some fast chasers, Physical Review E Statistical Nonlinear and Soft Matter Physics 86 (6 Pt 2) (2012) 067102.

[21] W. R. Siegfried, L. G. Underhill, Flocking as an anti-predator strategy in doves, Animal Behaviour 23 (75) (1975) 504-508.

[22] S. S. R. J. Neill, J. M. Cullen, Experiments on whether schooling by their prey affects the hunting behaviour of cephalopods and fish predators, Journal of Zoology 172 (4) (1974) 549-569.

[23] B. C. R. Bertram, Living in groups: predators and prey, in: Evolutionary Computation, 2005. The 2005 IEEE Congress on, 1978, pp. 41 - 48 Vol.1.

[24] K. K. Ingram, A. Pilko, J. Heer, D. M. Gordon, Colony life history and lifetime reproductive success of red harvester ant colonies, Journal of Animal Ecology 82 (3) (2013) 540-550.

[25] D. M. Gordon, The rewards of restraint in the collective regulation of foraging by harvester ant colonies, Nature 498 (7452) (2013) 91.

[26] O. T. Eldakar, M. J. Dlugos, J. W. Pepper, D. S. Wilson, Population structure mediates sexual conflict in water striders, Science 326 (5954) (2009) 816-816.

[27] L. Angelani, Collective predation and escape strategies, Physical Review Letters 109 (11) (2012) 118104.

[28] C. S. Holling, The functional response of invertebrate predators to prey density1, Memoirs of the Entomological Society of Canada 98 (48) (1966) 1-86.

[29] J. M. Jeschke, M. Kopp, R. Tollrian, Predator functional responses: discriminating between handling and digesting prey, Ecological Monographs 72 (1) (2002) 95-112.

[30] A. M. Wilson, T. Y. Hubel, S. D. Wilshin, J. C. Lowe, M. Lorenc, O. P. Dewhirst, H. L. Bartlam-Brooks, R. Diack, E. Bennitt, K. A. Golabek, et al., Biomechanics of predator-prey arms race in lion, zebra, cheetah and impala, Nature 554 (7691) (2018) 183.

[31] A. I. Houston, J. M. McNamara, J. M. Hutchinson, General results concerning the trade-off between gaining energy and avoiding predation, Phil. Trans. R. Soc. Lond. B 341 (1298) (1993) 375-397.

[32] S. A. Rands, G. Cowlishaw, R. A. Pettifor, J. M. Rowcliffe, R. A. Johnstone, Spontaneous emergence of leaders and followers in foraging pairs, Nature 423 (6938) (2003) 432.

[33] G. Beauchamp, G. D. Ruxton, False alarms and the evolution of antipredator vigilance, Animal Behaviour 74 (5) (2007) 1199-1206.

[34] C. W. Reynolds, Flocks, herds and schools: A distributed behavioral model, ACM SIGGRAPH Computer Graphics 21 (4) (1987) 25-34.

[35] V. Gaziy, K. M. Passinoz, A class of repulsion/attraction forces for stable swarm aggregations, International Journal of Control 18 (18) (2004) $1567-1579$

[36] R. Escobedo, C. Muro, L. Spector, R. P. Coppinger, Group size, individual role differentiation and effectiveness of cooperation in a homogeneous group of hunters, Journal of the Royal Society Interface 11 (95) (2014) 20140204. 